its upper, inner, and posterior surface there was a rupture of its surface large enough to admit a small crow's quill. This upon opening being enlarged was found to contain a cyst-like body about three fourths of an inch in diameter. This sac was quite firmly attached to the substance of the ovary, and upon being removed presented a velvety appearance externally.

When this sac was opened it was found filled with a clear watery fluid, with a kidney-shaped body about three eighths of an inch long floating in it, and seeming to be attached to its walls.

The sac and kidney-shaped body were examined by the naked eye alone, as the only condition upon which an autopsy could be obtained was by promising that nothing whatever should be carried away.

That this kidney-shaped body within the sac which was removed from the substance of the ovary was a foetus I have not the slightest doubt, but had it been examined carefully with a glass by an expert, and pronounced to be such, this confirmation would make the case a great deal stronger.

\title{
A CASE OF SOFTENING OF THE BRAIN.
}

BX EDWARD L. PARKs, M. D.

Av account of a case of brain softening, which ended a short time ago under my care at Mattapoisett, seems to me worthy of publication for several reasons, especially the youth of the patient, who was a young woman nineteen years of age, and the cause of her disease, - mental suffering and excitement dependent upon neglect and ill-treatment.

She was in childhood, according to her mother's statement, vigorous in mind and body. Her married sister and her brother are intelligent and healthy. Her life was somewhat irregular, and about two years ago she became pregnant by a young man who married her immediately upon the discovery of her condition. The child was born prematurely, after a fright, and did not survive. Dr. Sparrow attended her in her confinement, and about a year ago treated her for sleeplessness, which, he tells me, he relieved without difficulty. Some time after marriage her husband visited California, whence he returned with a gonorrhœa, and this he afterwards charged his wife with having communicated to him. Though living in the same town he refused to visit her. Her mother graphically describes her mental state during the last two years by saying, "Ever since her marriage she has been plagued almost to death." She was treated not long ago by an irregular practitioner of New Bedford for "retroversion of the womb." I have been unable to find a family history of syphilis, alcoholism, or insanity, and I believe her to have been free from them, for until after she came under 
my observation, about ten days before death, she was never insane, though " not quite herself."

When called to visit her, early in the morning, I was told that she had not slept the previous week, and had taken very little food. Menstruation had ceased a few days before. She was restless to a degree, with hallucinations, and there were slight choreic movements of the right hand and foot. She recognized those about her, and answered questions correctly. Pupils widely dilated, pulse 120, small and weak. Formication and cutaneous hyperæsthesia distinctly marked. She was ordered to have immediately as much beef broth as she could be induced to take, and chloral, of which she received nearly a drachm during the forenoon without sleep. I wrote at the same time for a tonic of iron, quinine, and strychnia, and for bromide of potassium in solution, twenty grains to be taken every night at bedtime. At noon $\mathrm{I}$ gave hypodermically one fourth of a grain of morphia, and, word having been sent me that she slept, did not visit her again that day.

She was treated the first three days of my attendance by nourishing food, gentle alcoholic stimulation, and quietude, with opium and morphia given plenteously. She occasionally slept an hour or two at a time. I was hastily summoned early in the morning of the third day to find her maniacal. She was promptly quieted by inhalations of ether, and I decided to examine the womb carefully as soon as possible, to learn if there was any acute lesion therein to cause the insanity. The os was found to be highly congested and everted, and a sound entered with difficulty. Blood was taken freely by leeches, beside punctures, and scarification of the uterine canal, previously dilated. Nitrate of silver and hot astringent washes were afterwards applied. For about two days more the general treatment was unchanged, except that I was obliged to administer ether two or three times every twenty-four hours, especially early in the mornings and late in the afternoons. One afternoon her language suddenly became obscene, and the same phrases were repeated over and over again. The gravity of these symptoms induced me to seek professional advice, and Dr. F. H. Hooper, of New Bedford, kindly gave me much valuable assistance. I became assured that the case was hopeless as to saving life or sanity, and not dependent upon uterine disease.

Her family were unwilling to send her to an asylum unless I believed her curable. Frankly declaring my belief that her life would soon end, I consented to treat her at her home. She was fed by the mouth as long as possible with beef tea, ice-cream, and wine, and afterwards by the rectum. Morphia and ether were used with caution. Ice applied to the head was grateful. She died from asthenia, respiration becoming labored at the last.

Post-mortem examination was made twenty hours after death, in the 
presence of Dr. Sparrow. Emaciation extreme, eyes deeply sun n, veins prominent, but no diffused discoloration. The cranial and abdominal cavities were examined. Brain and membranes tightly packed in their case; membranes congested. They were removed with great difficulty, the dura mater at the base of the brain being very dense, and the brain so soft as to break down at the slightest touch. The bladder was full of urine, and congested. Womb in normal position. Two serous sacs as large as peas were observed in folds of broad ligament. Internal os constricted. Os tincæ and upper part of vagina blackened. Bowels distended with air, but otherwise nearly empty. Appendiculæ epiploicæ contained very little fat. Nothing else noteworthy about abdominal viscera. An examination of the spinal cord was not practicable. With this exception all the parts were examined which seemed to bear upon the case. The brain and membranes and uterus and appendages were removed for further examination, and were at once put into a mixture of alcohol and water, one part to three. The next day, on attempting to dissect the brain, it was found throughout to be so softened that its main anatomical relations were scarcely recognizable.

The uterus was two and a half inches long without, two inches within. Right ovary had at its summit a pouting cicatrix, and an incision through its substance showed Graafian vesicles nearly mature. The surface of the left ovary was smooth. It seems to me that in her case the ovaries acted alternately. The treatises on physiology which I have consulted are silent upon this subject, and I do not know whether there is any rule.

From the history of the case I am led to believe it to have been one of general red softening of the brain (Da Costa, Bauduy). The authors just quoted do not enter as fully as I should wish into the differences between red softening and atrophic or white softening, - brain necrosis of Niemeyer. Flint, in his systematic treatise on the Practice of Medicine, describes softening as Circumscribed and Inflammatory. But whatever the variety may have been, I am satisfied that the disease had made such progress when I was called to it that speedy death was inevitable.

\section{RECENT PROGRESS IN OTOLOGY.}

BY J. ORNE GREEN, M. D.

Inspection of the Naso-Pharynx from the Nostrils. - Although the inspection of the nose and naso-pharyngeal cavity through the nostrils has been used to a limited extent for many years, it has never obtained a very extended usefulness except in diseases of the anterior third of

1 Concluded from page 504. 